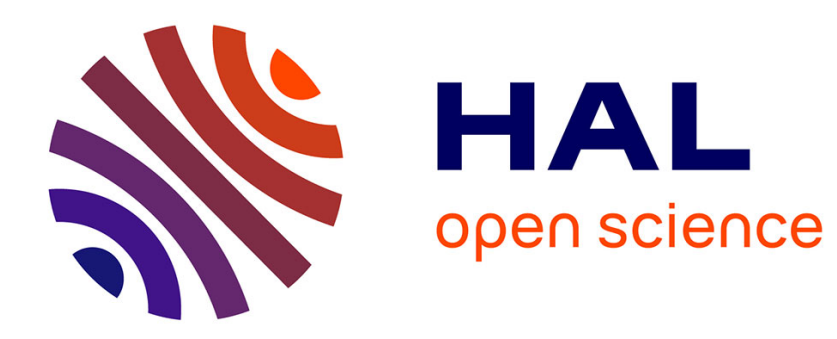

\title{
Résolution des équations de l'effet Kirkendall
}

J.F. Cornet

\section{To cite this version:}

J.F. Cornet. Résolution des équations de l'effet Kirkendall. Revue de Physique Appliquée, 1973, 8 (4), pp.293-305. 10.1051/rphysap:0197300804029300 - jpa-00243686

\section{HAL Id: jpa-00243686 https://hal.science/jpa-00243686}

Submitted on 1 Jan 1973

HAL is a multi-disciplinary open access archive for the deposit and dissemination of scientific research documents, whether they are published or not. The documents may come from teaching and research institutions in France or abroad, or from public or private research centers.
L'archive ouverte pluridisciplinaire HAL, est destinée au dépôt et à la diffusion de documents scientifiques de niveau recherche, publiés ou non, émanant des établissements d'enseignement et de recherche français ou étrangers, des laboratoires publics ou privés. 


\title{
REVUE DE PHYSIQUE APPLIQUÉE
}

\author{
Supplément au "Journal de Physique *
}

Classification

Physics Abstracts

7.630

\section{RÉSOLUTION DES ÉQUATIONS DE L'EFFET KIRKENDALL}

\section{J. F. CORNET}

Section de Métallurgie du Plutonium et des Actinides, Centre d'Etudes Nucléaires, Bât. 19, B. P. no 6, 92260 Fontenay-aux-Roses, France

(Reçu le 8 janvier 1973)

\begin{abstract}
Résumé. - Les propriétés de l'effet Kirkendall, déduites des équations classiques de Darken, sont vérifiées et développées dans différents cas de figure à l'aide de programmes de calcul numérique $(*)$ (Fortran).

Les calculs mettent en évidence une instabilité dans le déplacement du plan de Kirkendall, due à la discontinuité de la déformation à son voisinage.

Cette instabilité explique les nombreux ennuis rencontrés dans les expériences, et jusqu'ici attribués à des phénomènes parasites : fragmentation des repères, dispersion dans les déplacements. Un exemple de fragmentation est analysé quantitativement.
\end{abstract}

\begin{abstract}
The features of the Kirkendall effect, deduced from Darken's equations have been developped and verified for various cases with the aid of a Fortran, programmed computer. The calculation predicts an instability of the displacement of the Kirkendall plane caused by a discontinuity in the deformation in its neighborhood.

This instability explains a number of problems encountered in Kirkendall type experiments, problems which had hitherto been attributed to non-intrinsic-effects : marker fragmentation and large scatter in the measurements of the displacements. An example of marker fragmentation is analysed quantitatively.
\end{abstract}

Introduction. - L'effet Kirkendall est un phénomène facile à saisir d'une façon intuitive : deux éléments $\mathrm{A}$ et $\mathrm{B}$ diffusant dans un réseau cristallin n'ont pas la même vitesse moyenne. Il en résulte au cours de l'homogénéisation une déformation de ce réseau puisque certaines régions voient disparaître des atomes (effondrement de plans cristallins), tandis que d'autres en voient apparaître (création de plans cristallins).

Malheureusement les équations mises en jeu sont quasiment insolubles d'un point de vue analytique, même avec les hypothèses simplificatrices extrêmes (géométrie unidirectionnelle et nombres de sites par unité de volume constant).

Nous allons présenter deux programmes de calcul. Le premier a pour but de prévoir quantitativement l'importance du phénomène dans un couple de diffusion chimique, si l'on connaît les coefficients « intrinsèques " $D_{\mathrm{A}}$ et $D_{\mathrm{B}}$. Le second est un programme d'interprétation des données expérimentales, qui permet d'accéder aux fonctions $D_{\mathrm{A}}$ et $D_{\mathrm{B}}$ lorsqu'on a mesuré dans le couple le profil de concentration et la déformation induite par effet Kirkendall.

$\left(^{*}\right)$ Une liste des instructions Fortran est à la disposition du lecteur qui en fera la demande à :

SMPuA, Bt 19 CEN/FAR, BP 6, 92260 Fontenay-aux-Roses.
Nous allons tout d'abord rappeler brièvement les équations qui régissent le phénomène; puis nous présenterons les programmes avec quelques exemples d'applications numériques, en insistant sur les propriétés jusqu'à présent ignorées des expérimentateurs.

1. Rappels. - 1.1 HyPOTHèses ET DÉFINITIONS. 1.1.1 Concentrations. - Soit un couple de diffusion $\mathrm{AB} / \mathrm{BA}$, dans lequel nous regardons évoluer la concentration atomique $N_{\mathrm{A}}$ définie comme le rapport

$$
N_{\mathrm{A}}=\frac{n_{\mathrm{A}}}{n}
$$

où $n_{\mathrm{A}}$ est le nombre d'atomes $\mathrm{A}$ par unité de volume, $n$ le nombre total de sites par unité de volume.

Nous définirons de même la concentration

$$
N_{\mathrm{B}}=\frac{n_{\mathrm{A}}}{n}
$$

avec

$$
n_{\mathrm{A}}+n_{\mathrm{B}}=n, \quad N_{\mathrm{A}}+N_{\mathrm{B}}=1 .
$$

Deux hypothèses sont essentielles à la suite des calculs :

$1^{\circ} n$, nombre de sites par unité de volume, ne dépend pas de la concentration. 
$2^{\circ} n_{\mathrm{A}}+n_{\mathrm{B}}=n$, c'est-à-dire que tous les sites cristallins sont occupés. Ceci revient à négliger le nombre des sites vacants (lacunes) et le nombre des sites en excès (interstitiels).

Cette approximation est justifiée dans un métal proche de l'état d'équilibre.

Cependant l'effet Kirkendall s'accompagne presque toujours de porosités dont il est difficile de tenir compte dans les calculs.

1.1.2 Flux (voir remarque en Annexe II). - En chaque point du couple de diffusion il y a transport d'atomes A et B.

Soit $J_{\mathrm{A}}$ le nombre d'atomes A traversant l'unité de surface perpendiculaire à la direction de diffusion pendant l'unité de temps, $J_{\mathbf{B}}$ le nombre d'atomes $B$, dans les mêmes conditions. En général

$$
J_{\mathrm{A}}+J_{\mathrm{B}} \neq 0 \text {. }
$$

Il en résulte, si l'on veut que le nombre de sites par unité de volume soit constant, une déformation locale et un phénomène de translation.

a) Phénomène de translation.

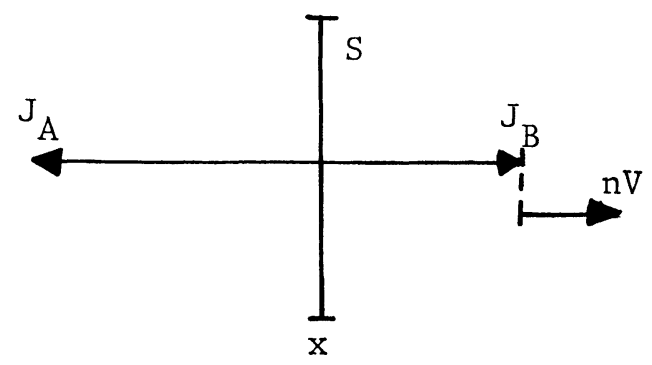

La surface $S$ située à l'abscisse $x$ voit passer pendant le temps $\mathrm{d} t$ :

$$
\mathrm{d} n=J_{\mathrm{A}} \cdot S \mathrm{~d} t+J_{\mathrm{B}} S \mathrm{~d} t \text { atomes } .
$$
de :

Elle devra donc se déplacer pendant ce même temps

$$
\mathrm{d} x=V \mathrm{~d} t=\frac{\mathrm{d} n}{n \cdot S}=-\frac{J_{\mathrm{A}}+J_{\mathrm{B}}}{n} \mathrm{~d} t .
$$

La vitesse de déplacement sera donc en tout point :

$$
-V=\frac{J_{\mathrm{A}}+J_{\mathrm{B}}}{n}
$$

b) Déformation. - Soit un volume $\Omega=\left(x^{\prime}-x\right) . S$.

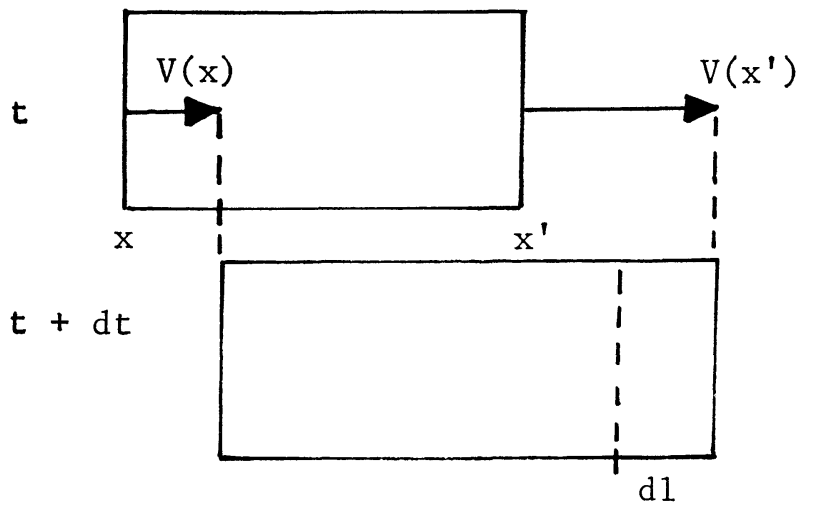

Le nombre total d'atomes à l'intérieur du volume $\Omega$ varie pendant le temps d $t$ de la quantité

$$
\mathrm{d} n=\left(J_{\mathrm{A}}\left(x^{\prime}\right)-J_{\mathrm{A}}(x)+J_{\mathrm{B}}\left(x^{\prime}\right)-J_{\mathrm{B}}(x)\right) S \mathrm{~d} t .
$$

La distance $l=x^{\prime}-x$ varie donc de $\mathrm{d} l=\mathrm{d} n / n S$

$$
\mathrm{d} l=\frac{1}{n}\left(J_{\mathrm{A}}\left(x^{\prime}\right)-J_{\mathrm{A}}(x)+J_{\mathrm{B}}\left(x^{\prime}\right)-J_{\mathrm{B}}(x)\right) \mathrm{d} t .
$$

Faisons tendre $x^{\prime}$ vers $x$ avec $x^{\prime}-x=l=\mathrm{d} x$ :

$$
\mathrm{d} l=\frac{1}{n} \frac{\partial}{\partial x}\left(J_{\mathrm{A}}+J_{\mathrm{B}}\right) \cdot l \cdot \mathrm{d} t .
$$

D'où la vitesse de déformation :

$$
\frac{1}{l} \frac{\mathrm{d} l}{\mathrm{~d} t}=\frac{\partial V}{\partial x} \text {. }
$$

1.2 BILAN DES FLUX. - Considérons un élément de volume $S \mathrm{~d} x$ à l'instant $t$. Il contient $n N_{\mathrm{A}}(t) S \mathrm{~d} x$ atomes $\mathrm{A}$.

A l'instant $t+\mathrm{d} t$ la concentration $N_{\mathrm{A}}$ a varié :

$$
\left.N_{\mathrm{A}}^{\prime}=N_{\mathrm{A}}+\frac{\partial N_{\mathrm{A}}}{\partial t}\right]_{x} \mathrm{~d} t
$$

et l'élément de volume s'est déformé, pour donner :

$$
d x^{\prime}=d x+\frac{\partial V}{\partial x} d x d t
$$

Le nombre total d'atomes qu'il contient maintenant est donc :

$$
\begin{aligned}
& \left.n S\left(N_{\mathrm{A}}+\frac{\partial N_{\mathrm{A}}}{\partial t}\right]_{x} \mathrm{~d} t\right)\left(\mathrm{d} x+\frac{\partial V}{\partial x} \mathrm{~d} x \mathrm{~d} t\right) \simeq \\
& \left.\simeq n N_{\mathrm{A}} S \mathrm{~d} x+n N_{\mathrm{A}} S \frac{\partial V}{\partial x} \mathrm{~d} x \mathrm{~d} t+n S \frac{\partial N_{\mathrm{A}}}{\partial t}\right]_{x} \mathrm{~d} x \mathrm{~d} t
\end{aligned}
$$

L'accroissement positif ou négatif du nombre d'atomes de l'espèce $\mathrm{A}, S N_{\mathrm{A}}$, comprend deux termes :

$\left.1^{\text {o Le terme }} n S \frac{\partial N_{\mathrm{A}}}{\partial t}\right]_{x} \mathrm{~d} x \mathrm{~d} t$ dû à l'évolution de la concentration.

$2^{0}$ Le terme $n S N_{\mathrm{A}} \frac{\partial V}{\partial x} \mathrm{~d} x \mathrm{~d} t$ dû à la dilatation.

D'après la définition du flux de diffusion $J_{\mathrm{A}}$, cet accroissement doit être égal à :

$$
-\frac{\partial J_{\mathrm{A}}}{\partial x} S \mathrm{~d} x \mathrm{~d} t
$$

Donc :

$$
\left.\frac{\partial N_{\mathrm{A}}}{\partial t}\right]_{x}+N_{A} \frac{\partial V}{\partial x}=-\frac{1}{n} \frac{\partial J_{\mathrm{A}}}{\partial x}
$$

Mais ce bilan ne tient pas compte du déplacement de l'élément de volume par rapport aux extrémités du couple de diffusion : l'abscisse $x$ choisie est celle du référentiel cristallin. Dans le référentiel du laboratoire nous avons l'abscisse $X$ :

$$
\mathrm{d} X=\mathrm{d} x+V \mathrm{~d} t
$$


d'où

$$
\left.\left.\frac{\partial N_{\mathrm{A}}}{\partial t}\right]_{X}=\frac{\partial N_{\mathrm{A}}}{\partial t}\right]_{x}-V \frac{\partial N_{\mathrm{A}}}{\partial X}
$$

De la relation (3) nous déduisons alors :

$$
\left.-\frac{\partial N_{\mathrm{A}}}{\partial t}\right]_{X}=\frac{1}{n} \frac{\partial J_{\mathrm{A}}}{\partial X}+\frac{\partial\left(N_{\mathrm{A}} V\right)}{\partial X}
$$

et en tenant compte de (1) :

$$
\left.\frac{\partial N_{\mathrm{A}}}{\partial t}\right]_{X}=-\frac{1}{n}\left[\frac{\partial J_{\mathrm{A}}}{\partial X}-\frac{\partial\left(\left(J_{\mathrm{A}}+J_{\mathrm{B}}\right) N_{\mathrm{A}}\right)}{\partial X}\right]
$$

ou

$$
\left.\frac{\partial N_{\mathrm{A}}}{\partial t}\right]_{X}=-\frac{1}{n} \frac{\partial}{\partial X}\left(N_{\mathrm{B}} J_{\mathrm{A}}-N_{\mathrm{A}} J_{\mathrm{B}}\right) .
$$

Jusqu'à présent nous n'avons émis aucune hypothèse physique sur le « moteur » de la diffusion.

Nous allons maintenant introduire les lois de Fick.

1.3 Lor DE FICK. - Si la diffusion se fait en l'absence de forces extérieures (champ électrique, gradient de température, ...), les flux $J_{\mathrm{A}}$ et $J_{\mathrm{B}}$ sont proportionnels aux gradients de concentration

$$
\begin{aligned}
& \frac{\partial N_{\mathrm{A}}}{\partial X} \text { et } \frac{\partial N_{\mathrm{B}}}{\partial X}: \\
& J_{\mathrm{A}}=-n D_{\mathrm{A}} \frac{\partial N_{\mathrm{A}}}{X} \\
& J_{\mathrm{B}}=-n D_{\mathrm{B}} \frac{\partial N_{\mathrm{B}}}{\partial X} .
\end{aligned}
$$

L'équation de conservation, improprement appelée « deuxième loi de Fick» devient alors :

$$
\left.\frac{\partial N_{\mathrm{A}}}{\partial t}\right]_{X}=+\frac{\partial}{\partial X}\left[\left(N_{\mathrm{A}} D_{\mathrm{B}}+N_{\mathrm{B}} D_{\mathrm{A}}\right) \frac{\partial N_{\mathrm{A}}}{\partial X}\right] .
$$

Les coefficients $D_{\mathrm{A}}$ et $D_{\mathrm{B}}$ sont supposés ne dépendre que de la concentration $N_{\mathrm{A}}=1-N_{\mathrm{B}}$.

Nous voyons l'intérêt de définir un nouveau coefficient $\widetilde{D}=N_{\mathrm{A}} D_{\mathrm{B}}+N_{\mathrm{B}} D_{\mathrm{A}}$ qui lui aussi ne dépend que de $N_{\mathrm{A}}$ et dont la connaissance suffit à déterminer le profil de concentration.

Chronologiquement il semble que le coefficient $\widetilde{D}$ ait été défini et mesuré avant les coefficients $D_{\mathrm{A}}$ et $D_{\mathrm{B}}$, ce qui amène souvent une confusion dans l'exposé de la théorie.

Tout se passe dans le référentiel du laboratoire comme si la diffusion était gouvernée par ce coefficient $\widetilde{D}$ apparent, qui masque la différence de diffusivité des deux espèces, et interdit l'accès aux coefficients intrinsèques $D_{\mathrm{A}}$ et $D_{\mathrm{B}}$, par une simple mesure de profil de concentration.

1.4 RéSOlution Des ÉQuATIONS De DARKen. - La mesure du profil de concentration $N_{\mathrm{A}}(X, t)$ d'un couple de diffusion ne permet pas d'accéder aux coefficients intrinsèques $D_{\mathrm{A}}$ et $D_{\mathrm{B}}$.
Pour y accéder, il faut mesurer en plus la déformation qui a lieu dans le couple pendant la durée du recuit, ou le déplacement de repères placés initialement dans la zone de diffusion.

Nous avons vu [2] que ce déplacement est localement lié aux coefficients de diffusion intrinsèques par la relation :

$$
X-X_{0} \frac{\partial X}{\partial X_{0}}=2 t\left(D_{\mathrm{A}}-D_{\mathrm{B}}\right) \frac{\partial N_{\mathrm{A}}}{\partial X}
$$

Cette expression résulte directement de celle de la vitesse $(\S 1.1 .2 a)$ :

$$
V=-\frac{J_{\mathrm{A}}+J_{\mathrm{B}}}{n}=\left(D_{\mathrm{A}}-D_{\mathrm{B}}\right) \frac{\partial N_{\mathrm{A}}}{\partial X} .
$$

Les programmes de calcul numérique que nous. proposons permettent de résoudre le système d'éq. (6) et (7) dans les deux cas :

$1^{\circ}$ On se donne $D_{\mathrm{A}}$ et $D_{\mathrm{B}}$ pour toute valeur de $N_{\mathrm{A}}$. On veut calculer $N_{\mathrm{A}}(X, t)$ et $X\left(X_{0}, t\right)$.

$2^{0}$ On se donne $N_{\mathrm{A}}(X)$ et $X\left(X_{0}\right)$ à un instant $t$. On veut calculer $D_{\mathrm{A}}$ et $D_{\mathrm{B}}$.

1.5 Les équations utiles auX CalCuls - Il y a tout d'abord trois grandeurs qui jouent un rôle prépondérant dans les différentes étapes; ce sont :

- la variable réduite $\lambda=X / \sqrt{t}$ car on sait que la concentration $N_{\mathrm{A}}$ ne dépend que de cette variable ;

- le coefficient $\widetilde{D}=N_{\mathrm{A}} D_{\mathrm{B}}+N_{\mathrm{B}} D_{\mathrm{A}}$, que l'on pourrait qualifier de coefficient de diffusion «apparent », et auquel on peut accéder par les mesures de la concentration $N_{\mathrm{A}}$;

- la fonction

$$
F(\lambda)=2\left(D_{\mathrm{A}}-D_{\mathrm{B}}\right) \frac{\mathrm{d} N_{\mathrm{A}}}{\mathrm{d} \lambda},
$$

qui a la même dimension que $\lambda\left(\mathrm{cm} . \mathrm{s}^{-1 / 2}\right)$ et qui gouverne l'effet Kirkendall. On peut y accéder directement et sans mesure de concentration pourvu que l'on connaisse la déformation du couple en tout point à un instant donné.

Le calcul du coefficient de diffusion chimique $\widetilde{D}\left[N_{\mathrm{A}}(x, t)\right]$ se fait point par point par la méthode de Matano

$$
\tilde{D}=-\frac{1}{2 t} \frac{\int_{N_{0}}^{N_{\mathrm{A}}} X \mathrm{~d} N_{\mathrm{A}}}{\partial N_{\mathrm{A}} / \partial X} .
$$

Connaissant $\widetilde{D}$ on peut revenir à $N_{\mathrm{A}}(x, t)$ par la méthode itérative Matano $^{-1}$ [1].

La fonction $F$ sera déterminée à l'aide de la relation (9)

$$
X-X_{0} \frac{\partial X}{\partial X_{0}}=F \sqrt{t}
$$


Pour le calcul inverse, on dispose de l'équation différentielle :

$$
\frac{\mathrm{d} X}{\mathrm{~d}(\sqrt{t})}=F
$$

que l'on peut résoudre numériquement par la méthode du tir ; on dispose également de son intégrale :

$$
X_{0}=X_{1} \exp \int_{\lambda_{1}}^{\lambda} \frac{\mathrm{d} \lambda}{\lambda-F}
$$

qui se calcule en deux parties, pour les intervalles

$$
[-\infty, 0] \text { et }[0,+\infty] \text {. }
$$

2. Problème direct : programme Effet K. - Les coefficients intrinsèques de chacune des espèces, $D_{\mathrm{A}}$ et $D_{\mathrm{B}}$, sont supposés connus.

Ces fonctions pourront être données sous forme de tableaux :

- tableau du coefficient $D_{\mathrm{A}}$,

- tableau du coefficient $D_{\mathrm{B}}$,

- tableau des concentrations correspondantes $C$;

ou sous forme de sous-programmes du type Function si l'on dispose d'une formule empirique pour la variation des coefficients avec la concentration : la figure 1 présente un organigramme sommaire des calculs.

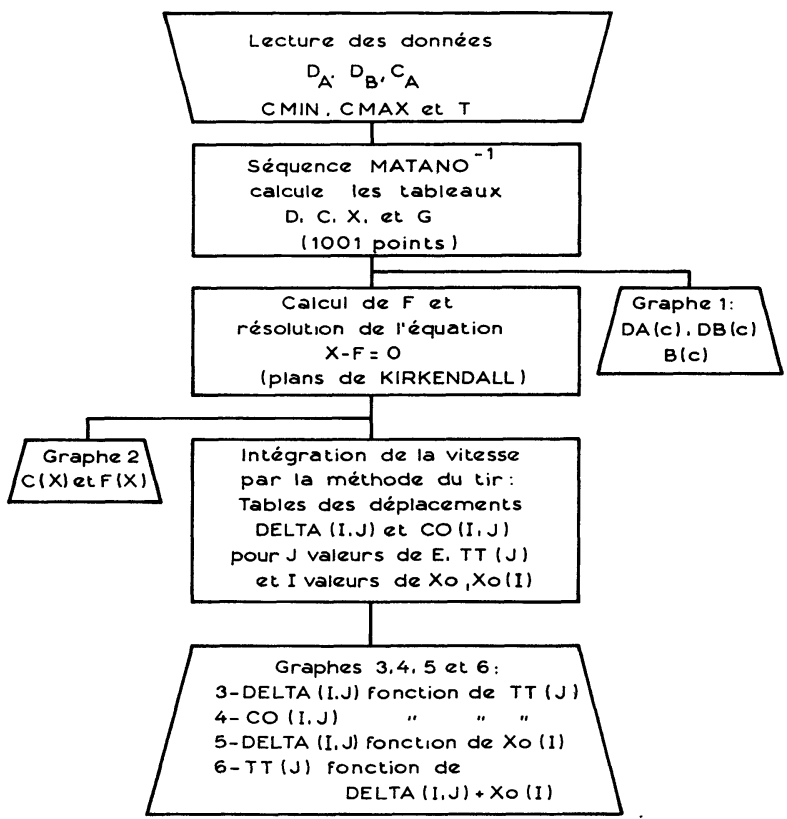

FIG. 1. - Organigramme du calcul des profils de concentration et de déplacements à partir des coefficients de diffusion intrinsèque (effet K).

2.1 Première MÉTHOde - Le programme calcule :

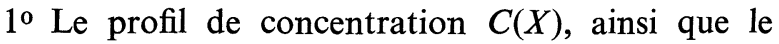
gradient de concentration $G$. $X$ désigne dans le programme la variable réduite $\lambda, C$ la concentration $N_{\mathrm{A}}$ et $G$ la quantité $\mathrm{d} N_{\mathrm{A}} / \mathrm{d} \lambda$.

Les tableaux $X, C$ et $G$ comprennent chacun 1001 points. $2^{\circ}$ Le déplacement Delta $(I, J)$ et la concentration $C O(I, J)$ de 101 repères placés à l'instant zéro dans la zone de diffusion, à des intervalles égaux (abscisses $X O(I)$ ), pour 101 durées successives $T T(J)$ (échelle en racine carrée du temps).

2.1.1 Calcul du profil de concentration. - Le programme Matano $^{-1}$ a été amélioré par rapport à la version décrite en [1] ; au lieu de maintenir fixe le tableau des abscisses $X$ et de calculer à chaque itération les nouvelles valeurs des concentrations $C$ correspondantes, on fixe cette fois le tableau des concentrations et l'on fait varier les valeurs de l'abscisse.

Ce nouveau procédé élimine l'inconvénient majeur de la $1^{\text {re }}$ version : choix d'un intervalle pour les abscisses ; l'intervalle étant volontairement trop grand (s'il est trop petit les conditions aux limites ne sont pas respectées), un nombre important de points se trouve hors de la zone de diffusion donc ne sert à rien.

La figure 2 donne un organigramme sommaire de la séquence corrigée. Cette nouvelle version n'est pas plus coûteuse que la précédente, ni en temps de calcul, ni en encombrement ; en effet, si elle oblige à recalculer le tableau des abscisses à chaque itération, elle évite d'avoir à recalculer les valeurs des coefficients de diffusion puisque les concentrations ne varient pas.

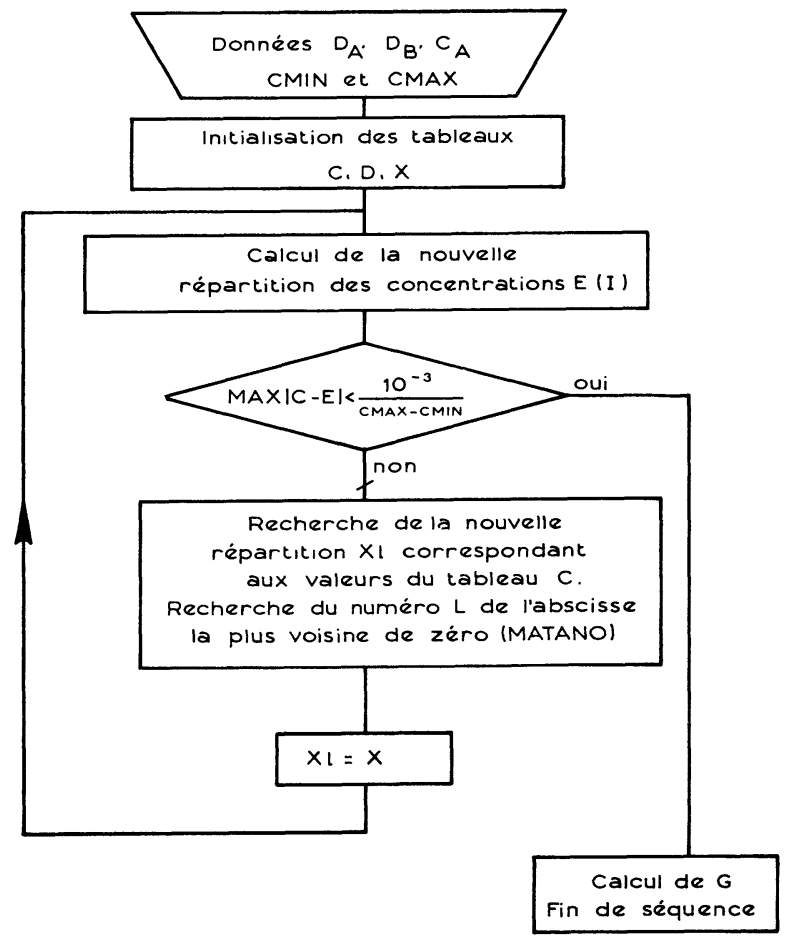

Fig. 2. - Organigramme du calcul des profils de concentration $N_{\mathrm{A}}(\lambda)$ à partir du coefficient de diffusion chimique (Matano-1).

Les résultats de cette première séquence sont placés dans les tableaux :

- $X$ abscisses (variable réduite),

- $C$ concentrations,

- $D$ coefficients de diffusion,

- $G$ gradients de concentration (variable réduite). 
Sur 1001 points, l'intervalle de concentration étant divisé en 1000 intervalles égaux.

2.1.2 Calcul du déplacement des repères et des concentrations correspondantes. - Cette phase comporte :

$1^{0}$ Calcul de la quantité

$$
F=2 *\left(D_{\mathrm{A}}-D_{\mathrm{B}}\right) * G,
$$

pour chacun des 1001 points correspondant au tableau des concentrations.

$2^{\circ}$ Résolution de l'équation

$$
X-F=0
$$

les solutions $X_{\mathbf{K}}$ sont les abscisses correspondant à l'interface initial (interfaces de Kirkendall).

Le programme calcule les concentrations correspondantes $C_{\mathrm{K}}$.

$3^{\circ}$ Calcul du déplacement de 101 repères placés à l'instant zéro dans un intervalle correspondant à la zone de diffusion au temps $T . T$ figure en donnée du programme.

Pour chacun des repères on résout l'équation différentielle du mouvement :

$$
\mathrm{d}\left(X_{1}\right)=F \mathrm{~d}(\sqrt{t})
$$

où $X_{1}$ désigne la position $(\mathrm{en} \mathrm{cm}$ ) du repère à l'instant $t$. La durée $\sqrt{T}$ est divisée en 1001 intervalles $\Delta(\sqrt{t})$. La résolution se fait par la méthode du tir ; pour le $k$-ième intervalle de temps la position du repére $X_{1_{k}}$ est calculée par la récurrence :

$$
X_{1_{k}}=X_{1_{k-1}}+F\left(X_{1_{k-1}}\right) \Delta(\sqrt{t}) .
$$

On retient au cours des calculs un point sur dix c'est-à-dire la position du repére pour 101 valeurs successives de $\sqrt{t}$, et la concentration correspondante (Tableaux Delta $(I, J), C O(I, J)$, correspondant aux abscisses initiales $X O(I)$ et aux durées $T T(J))$.

2.1.3 Entrées-sorties. - Il faut fournir au programme principal :

- soit les tableaux $D_{\mathrm{A}}$ et $D_{\mathrm{B}}$ ainsi que les valeurs correspondantes de la concentration $C$,

- soit un sous-programme de calcul de ces coefficients,

- la durée du recuit de diffusion $T$,

- l'intervalle de concentration $C_{\min }, C_{\max }$ (cas des couples incrémentaux).

\subsubsection{Listing. - Le programme écrit :}

$1^{0}$ à la suite de la séquence Matano ${ }^{-1}$ un tableau comprenant 101 points; pour chaque point on peut lire l'abscisse $X\left(\mathrm{~cm} \cdot \mathrm{s}^{-1 / 2}\right)$, la concentration $C$, le coefficient de diffusion chimique $D$, le gradient de concentration $G$, et la quantité $F=2\left(D_{\mathrm{A}}-D_{\mathrm{B}}\right) \cdot G$;

$2^{\circ}$ la résolution de l'équation $F=G$ permet d'écrire l'abscisse du plan de Kirkendall (en valeur de $X / \sqrt{t}$ ), ainsi que l'incertitude sur sa détermination ; la concentration du plan de Kirkendall et l'incertitude sur sa détermination ; enfin sa durée nécessaire à un déplacement de $100 \mu$ en heures;

$3^{0}$ le calcul du déplacement des plans cristallins permet d'écrire, pour une vingtaine d'entre eux, la valeur de leur abscisse et la concentration correspondante en fonction de la racine carrée du temps.

2.1.3.2 Sorties graphiques. - Six graphes sont tracés, permettant de donner une image plus concrète du comportement des équations :

$1^{0}$ Graphe des coefficients de diffusion.

Les trois coefficients de diffusion $D_{\mathrm{A}}, D_{\mathrm{B}}$ et $\tilde{D}$ sont portés en fonction de la concentration.

$2^{\circ}$ Graphe, concentration, pénétration et courbe $F$.

Ce graphe permet de déterminer la position et la concentration du plan de Kirkendall. Il superpose la concentration et la fonction $2\left(D_{\mathrm{A}}-D_{\mathrm{B}}\right) \frac{\mathrm{d} C}{\mathrm{~d} X}$, la variable étant réduite $\left(\frac{X}{\sqrt{t}}\right)$. La position du plan de Matano est soulignée par un trait vertical; la droite $X=F$ est tracée. Elle n'est en général pas à $45^{\circ}$ car les échelles en $F$ et $X$ ne sont pas les mêmes. Là où les solution (s) de l'équation $X=F$ sont données par l'intersection de la droite et de la courbe $F$.

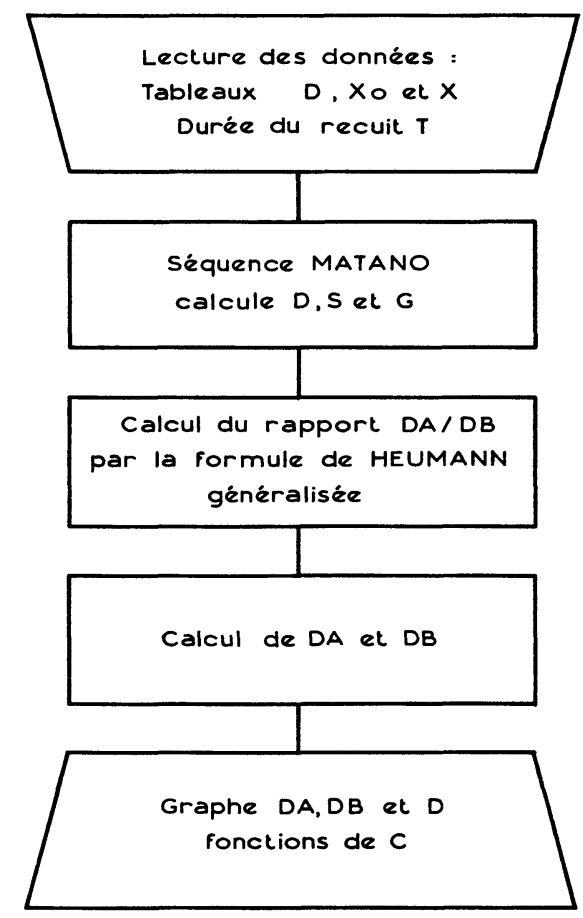

Fig. 3. - Organigramme du calcul des coefficients de diffusion intrinsèque à partir des profils de concentration et de déplacement.

$3^{\circ}$ Graphe du déplacement des repères avec la racine carrée du temps.

Pour une dizaine de repères, dont l'abscisse initiale est écrite en microns, on trace le déplacement $X-X_{0}$ 
(échelle en $\mathrm{cm}$ ) en fonction de la racine carrée du temps $\left(s^{1 / 2}\right)$.

$4^{0}$ Concentration des repères au cours du recuit.

Ce graphique est également porté en fonction de la racine carrée du temps, pour quelques repères placés de part et d'autre du plan de Kirkendall (abscisse initiale $=O . O$ ). $\mathrm{Ce}$ dernier a une concentration rigoureusement constante sauf pour les très petites valeurs de $\sqrt{ } t$; en effet il n'est pas possible de calculer numériquement la variable $\lambda=X / \sqrt{t}$ à l'instant zéro, même quand $X=0$, et il y a une petite imprécision sur la position de l'interface de collage. Nous verrons que cette imprécision qui est ici purement numérique, a également une interprétation physique ; en effet il n'existe pas de repère infiniment petit (un fil de tungstène mesure en général 10 à $15 \mu$ de diamètre). Il peut en résulter une grande imprécision dans le dépouillement des expériences.

$5^{\circ}$ Déplacement abscisse finale.

Pour cinq valeurs successives de la racine carrée du temps, on trace la courbe $X_{1}-X_{0}$ en fonction de $X_{1}$. Les valeurs de $X_{1}$ sont discrètes et parfois éloignées les unes des autres. Il en résulte que les graphes sont constitués de petits segments au voisinage du plan de Kirkendall, surtout pour les grandes valeurs de $\sqrt{t}$.

Ce réseau de courbes est homothétique, comme on peut le prévoir théoriquement [3].

De cette propriété on peut tirer une autre méthode de calcul beaucoup plus précise, sur laquelle nous reviendrons au paragraphe suivant (2.2).

$6^{0}$ Position des repères et racine carrée du temps.

En abscisse, on porte, pour chacun des 101 repères, sa position (même échelle que le graphe précédent). En ordonnée on porte la racine carrée du temps.

Ce graphe a pour but de mettre en évidence les variations de la distance relative entre repères voisins.

2.2 DEUXIÈme MÉTHODE. - Il s'agit en fait d'une variante du programme précédent qui utilise les équations décrites par ailleurs [3]. Le calcul du déplacement des repères se fait par la relation

$$
X_{0}=K \exp \int_{X_{1}}^{X} \frac{\mathrm{d} X}{X-F(X)}
$$

où $X$ représente la variable réduite $\lambda$, et $F$ la fonction :

$$
2\left(D_{\mathrm{A}}-D_{\mathrm{B}}\right) \frac{\partial N_{\mathrm{A}}}{\partial X}
$$

L'intégration se fait en deux parties ; d'abord pour $X<X_{k_{1}}$ avec une constante $K_{-}=X(1)$, puis pour $X>X_{k_{n}}$ avec une constante $K_{+}=X(N)$, car l'intégrale diverge chaque fois que $X=F$ (plan de Kirkendall). $X_{k_{1}}$ et $X_{k_{n}}$ sont respectivement la plus petite et la plus grande des solutions de l'équation $X=F$. Elles sont en général identiques.

On obtient ainsi les 1001 valeurs de $X_{0} / \sqrt{t}$ correspondant à l'instant $t$ aux 1001 valeurs de $X / \sqrt{ } t$.
Cette méthode de calcul est beaucoup plus précise que la précédente au voisinage du plan de Kirkendall puisque le tableau $X$ comprend 1001 points au lieu de 101 précédemment.

Elle met en évidence la discontinuité des pentes au voisinage du plan de Kirkendall, et permet de tracer en perspective la nappe $x-x_{0}$ fonction des deux variables $x$ et $\sqrt{t}$, qui comporte une arête de rebroussement [ou une discontinuité selon que le plan de Kirkendall est unique ou non].

2.3 EXEMPLes de RÉSUltats. - Afin d'observer le comportement des équations, nous avons soumis aux calculs un certain nombre de données «théoriques", c'est-à-dire ne correspondant pas à des systèmes connus expérimentalement.

Il est en effet extrêmement difficile de prévoir la forme de la courbe des déplacements de repères à partir des données de $D_{\mathrm{A}}$ et $D_{\mathrm{B}}$, même qualitativement. Les exemples choisis sont des cas typiques : cas de coefficients proportionnels $\left(D_{\mathrm{A}} / D_{\mathrm{B}}=\mathrm{Cte}\right)$, cas d'inversion $\left(D_{\mathrm{A}}-D_{\mathrm{B}}\right.$ change de signe), cas de plans multiples (l'équation $\lambda-F=0$ a plusieurs solutions). Dans l'état actuel de nos connaissances, tant théoriques qu'expérimentales, rien ne permet d'affirmer que telle ou telle configuration existe ou n'existe pas pour un système donné ; aucune formule théorique ni empirique n'est donnée à ce jour pour les variations des coefficients de diffusion intrinsèques avec la concentration ; très peu de systèmes ont été étudiés au point de vue de l'effet Kirkendall et en général les études ne portent pas sur toute la gamme des concentrations. Enfin l'imprécision des mesures (porosité, diamètre des repères), interdit toute spéculation sur le sens probable de variation des coefficients.

Les calculs nous permettront toutefois d'éclaircir en partie les obstacles expérimentaux qui se posent : dilatation de l'échantillon et porosité.

\subsubsection{Coefficients proportionnels. - La figure 4} montre la forme choisie pour les coefficients. Le rapport $D_{\mathrm{A}} / D_{\mathrm{B}}$ est égal à 10 . Le coefficient de diffusion chimique présente un maximum au voisinage de $N_{\text {A }}=0,6$.

La figure 5 présente le profil de concentration et la fonction $F$ en fonction de la variable réduite $\lambda$. La solution de l'équation $\lambda=F$ correspond à l'intersection de la $1^{\text {re }}$ bissectrice et la courbe $F$; ici pour la valeur $\lambda=-0,615 \times 10^{-4} \mathrm{~cm} . \mathrm{s}^{-1 / 2}$, ce qui correspond à une concentration voisine de 79,5\% (plan de Kirkendall). Nous remarquons que dans ce cas le maximum de la fonction $F$ (maximum de la vitesse $\mathrm{d} X / \mathrm{d} t$ en valeur absolue), coïncide avec le plan de Kirkendall. Ce n'est pas l'effet d'un hasard : on peut montrer (Annexe I) qu'une condition suffisante pour avoir une telle coïncidence est que le rapport $D_{\mathrm{A}} / D_{\mathrm{B}}$ soit constant quelle que soit la concentration.

Malheureusement il n'y a certainement pas proportionnalité entre les coefficients intrinsèques dans le cas 


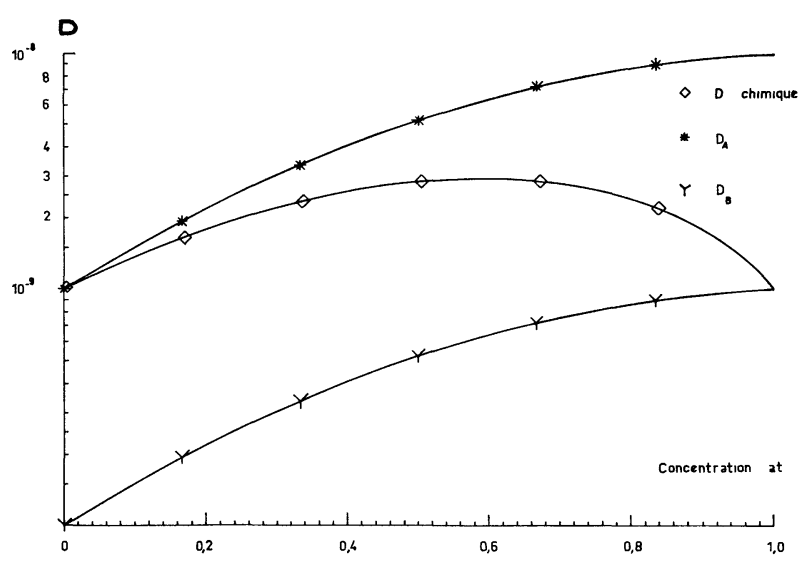

FIG. 4. - Coefficients de diffusion intrinsèques proportionnels et coefficient de diffusion chimique $\tilde{D}=N_{\mathrm{A}} \quad D_{\mathrm{B}}+N_{\mathrm{B}} \quad D_{\mathrm{A}}$ correspondant (la concentration $N_{\mathrm{A}}$ est portée en abscisse).

Fig. 4 à 9. - Les figures 4 à 9 correspondent aux différentes sorties graphiques du programme Effet $\mathrm{K}$ schématisé figure 1 .

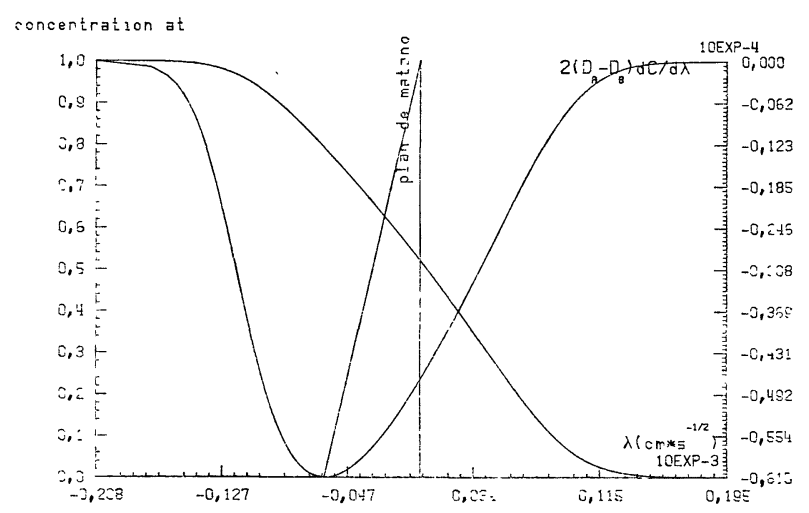

Fig. 5. - Profil de concentration $N_{\mathrm{A}}(\lambda)$ (échelle de gauche) et vitesse réduite $F(\lambda)$ (échelle de droite) correspondant aux coefficients de la figure 4 .

général ; cela impliquerait en particulier qu'aux extrémités de l'intervalle de concentration la loi soit également respectée, c'est-à-dire :

$$
\frac{D_{\mathrm{A}^{* \mathrm{~B}}}}{D_{\mathrm{B}^{* \mathrm{~B}}}}=\frac{D_{\mathrm{A}^{* \mathrm{~A}}}}{D_{\mathrm{B}^{* \mathrm{~A}}}}
$$

La figure 6 montre le réseau de courbes $X-X_{0}$, $\sqrt{t}$ pour différentes valeurs de l'abscisse initiale $X_{0}$.

Nous voyons que pour la valeur $X_{0}=0$ le déplacement est toujours proportionnel à $\sqrt{t}$ et que les lois empiriques du type :

$$
X=K\left(\sqrt{t}-\sqrt{t_{0}}\right) \quad \text { ou } \quad X=K \sqrt{t-t_{0}}
$$

ne sont pas respectées sauf lorsque la concentration du repère est suffisamment voisine de celle du plan de Kirkendall (la vitesse varie peu au voisinage de son maximum, dans ce cas).

La figure 7 montre l'évolution de la concentration des repères au cours du recuit. Nous voyons qu'elle tend vers la concentration du plan de Kirkendall dans tous les cas.

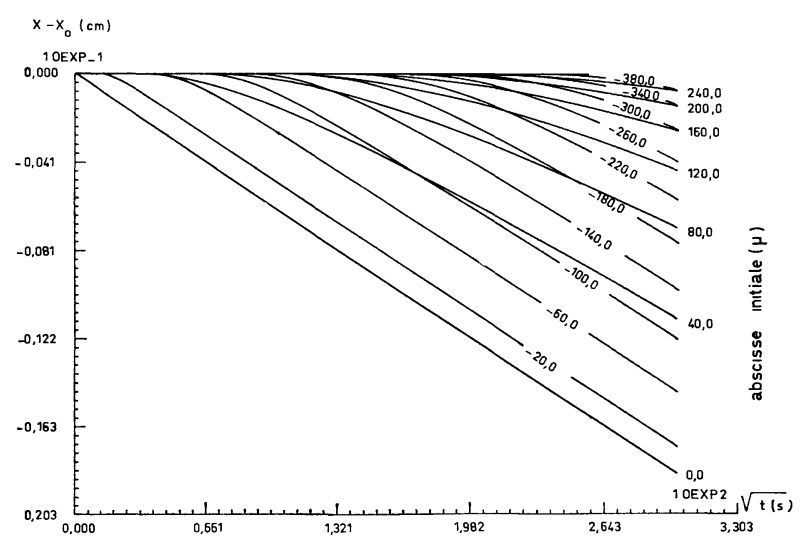

Fig. 6. - Déplacement des repères $X-X_{0}$ avec la racine carrée du temps dans le cas de la figure 4. L'abscisse initiale $X_{0}$ des repères est notée en microns par interruption de la courbe pour les valeurs $X_{0}$ négatives et en fin de courbe pour les valeurs positives.

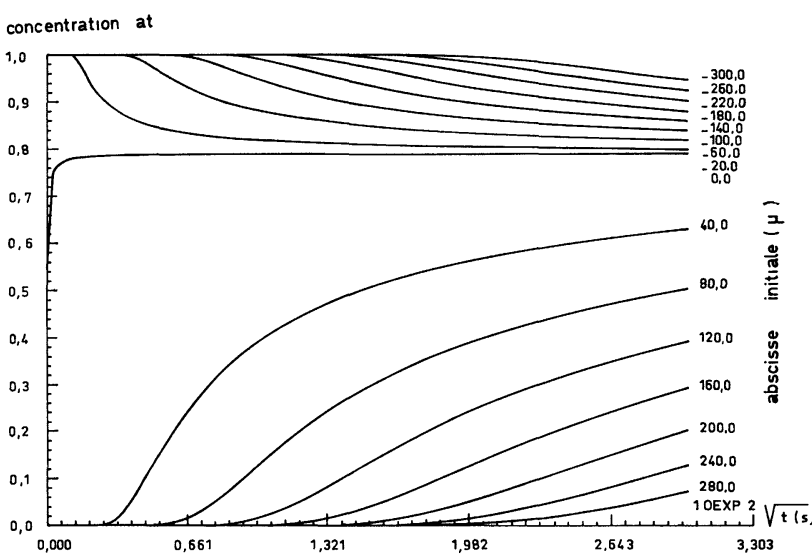

FIG. 7. - Concentration $N_{\mathrm{A}}$ au voisinage des repères (l'abscisse initiale est portée en fin de courbe) en fonction de $\sqrt{t}$, dans le cas de la figure 4.

La grille de la figure 8 présente pour les 101 repères l'abscisse en fonction de la racine carrée du temps. Nous voyons que les repères placés à gauche du plan de Kirkendall se rapprochent considérablement les uns des autres : disparition de plans cristallins.

De l'autre côté les repères s'écartent : apparition de plans.

La déformation au voisinage du plan de Kirkendall atteint ici des valeurs de l'ordre de $300 \%$ dès le début du recuit. Cette remarque confirme le mécanisme de formation des porosités ; en effet si pour les abscisses positives $\left(X_{0}>0\right)$ la déformation est une dilatation (création de plans), de l'autre côté c'est une contraction. Les mécanismes de diffusion imposent à deux plans cristallins sur trois de disparaître ; il est probable que la cinétique d'élimination des lacunes ne sera pas assez rapide pour réaliser cet effondrement; d'où l'apparition de porosité du côté du métal qui diffuse le plus vite au voisinage du plan de Kirkendall. 


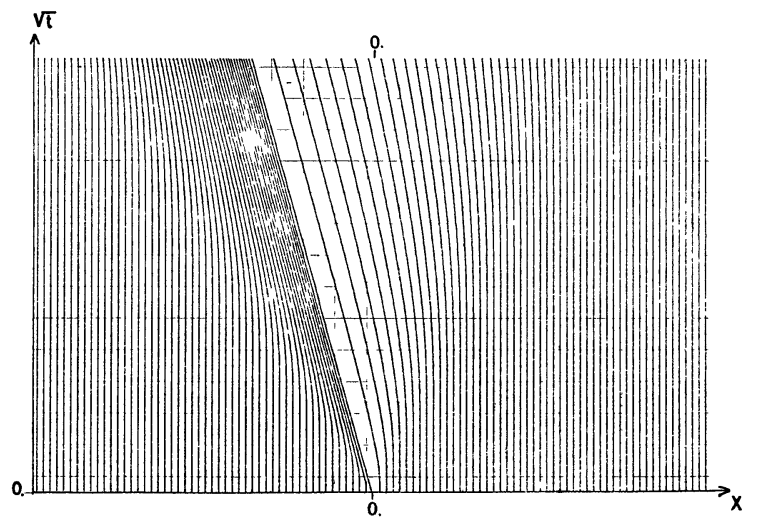

Fig. 8. - Diagramme des déplacements dans le cas de la figure 4. La racine carrée du temps est portée en abscisse, et la position des repères en ordonnée. La position initiale des repères peut être lue directement sur l'axe des abscisses $(\sqrt{t}=0)$ (échelles arbitraires) (101 repères).

De l'autre côté il y a une très importante dilatation. Le repère placé à l'interface du collage va être soumis à des tensions très importantes puisqu'il est inerte; il pourra éventuellement se rompre; si ce n'est pas le cas l'incertitude sur sa position finale sera égale à trois fois son diamètre puisque la déformation est de $300 \%$ (voir schéma, § 2.3.4).

Cela explique en partie la très mauvaise précision des mesures d'effet Kirkendall dans certains systèmes.

Enfin sur la figure 9 on peut voir les courbes de déplacement en fonction de l'abscisse. Elles présentent comme prévu un point anguleux pour l'abscisse du plan de Kirkendall.

Ces courbes sont par ailleurs homothétiques [3].

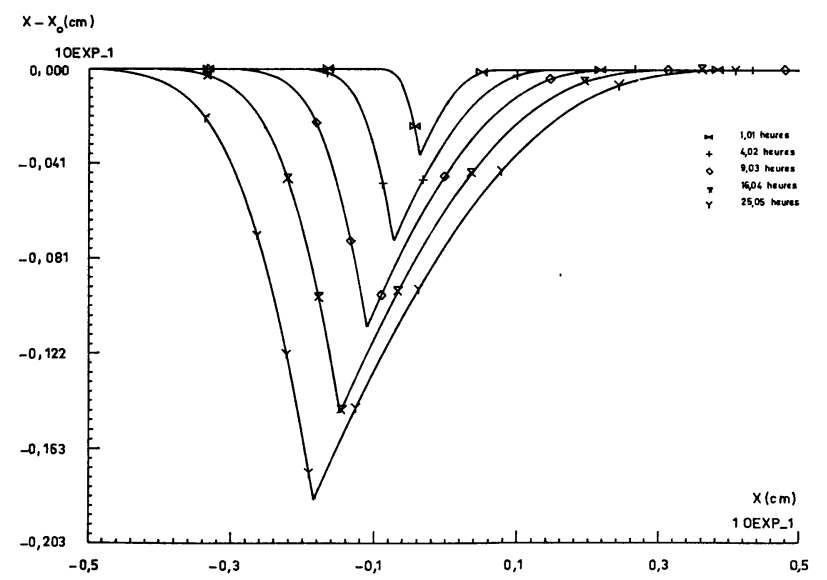

Fig. 9. - Profils de déplacements $X-X_{0}(X)$ correspondant au cas de la figure 4 , pour des durées de recuit variables.

2.3.2 Cas d'inversion. - Les figures 10 et 11 montrent un cas où $D_{\mathrm{A}}<D_{\mathrm{B}}$ dans l'intervalle de concentration compris entre $15 \%$ et $78 \%$. Le coefficient $\widetilde{D}$ accuse une légère inflexion et varie peu au milieu de l'intervalle.

La courbe $N_{\mathrm{A}}(\lambda)$ a une forme tout à fait régulière ; mais la fonction $F$ présente trois extréma et change deux fois de signe.

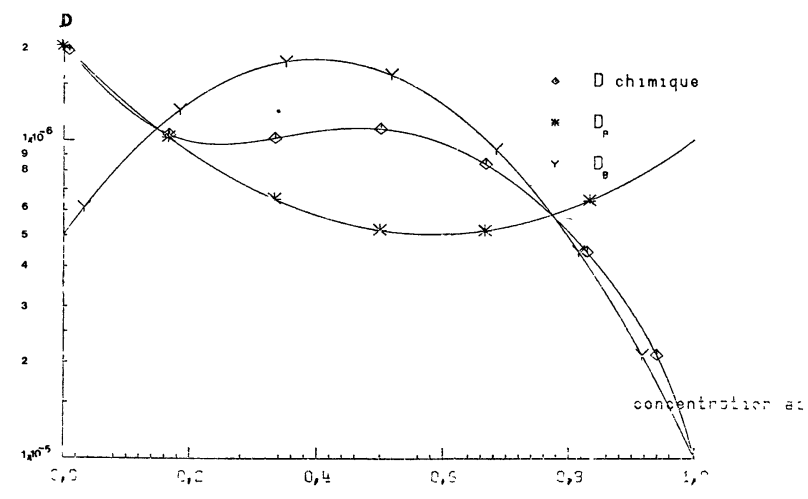

Fig. 10. - Coefficients de diffusion soumis aux calculs $D_{\mathrm{A}}\left(N_{\mathrm{A}}\right)$, $D_{\mathrm{B}}\left(N_{\mathrm{A}}\right)$ et $\tilde{D}\left(N_{\mathrm{A}}\right)$.

FIG. 10 à 13. - Les figures 10 à 13 sont les résultats d'un nouvel essai du programme schématisé figure 1 .

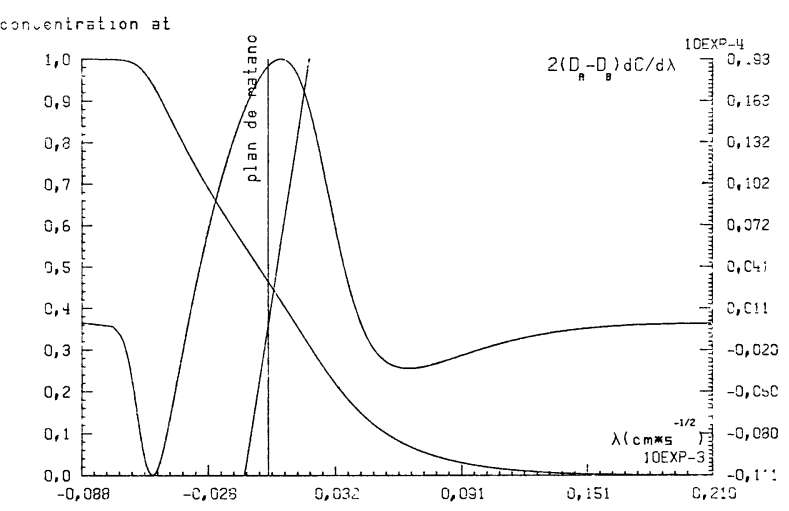

Fig. 11. - Profil $N_{\mathrm{A}}(\lambda)$ et vitesse réduite $F(\lambda)$ correspondant aux coefficients de la figure 10. Cas où $D_{\mathrm{A}}\left(N_{\mathrm{A}}\right)-D_{\mathrm{B}}\left(N_{\mathrm{B}}\right)$ change de signe.

Les figures 12 et 13 montrent les déplacements résultants.

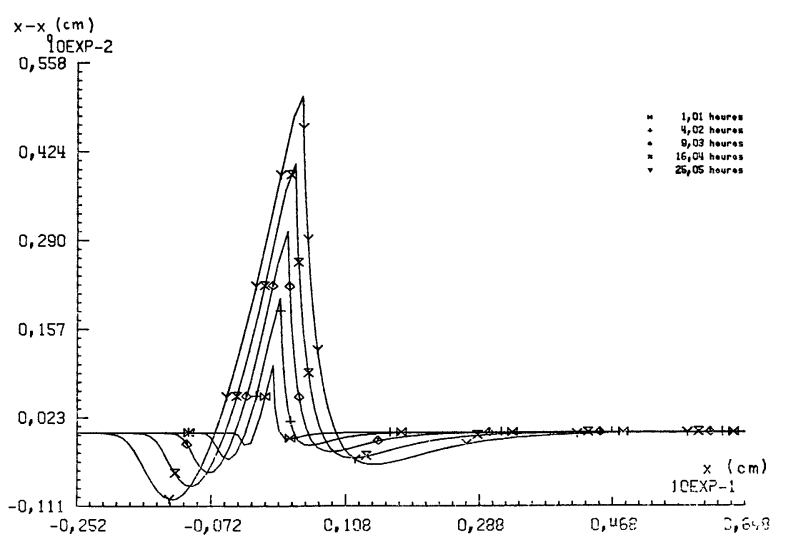

FIg. 12. - Profils de déplacements $\left(X-X_{0}\right)(X)$ pour cinq durées de diffusion.

Il existe toujours à un instant donné dans la zone deux repères dont le déplacement global est nul $\left(X-X_{0}=0\right)$.

Il existe également à tout instant deux repères dont la vitesse de déplacement est nulle $(F=0)$. 


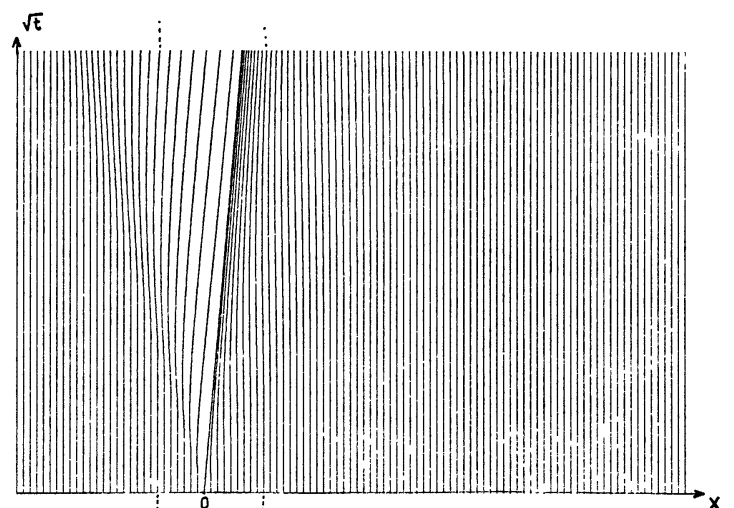

Fig. 13. - Diagramme des déplacements (mêmes remarques que pour la Fig. 8) avec les coefficients de la figure 10. L'échelle des abscisses n'est pas ici arbitraire ; elle correspond exactement à celle de la figure 12 . L'échelle en $\sqrt{t}$ correspond à la durée maximale ( $25 \mathrm{~h} 05)$ portée sur la figure 12.

Un cas d'inversion a été observé expérimentalement dans le système UZr [4], [5].

2.3.3 Cas de plans multiples. - La solution de l'équation $\lambda-F=0$ n'est pas nécessairement unique, bien que ce cas n'ait jamais été observé expérimentalement (Fig. 14, 15, 16, 17).

Les coefficients intrinsèques choisis sont symétriques par rapport à la concentration $N_{\mathrm{A}}=0,5$ (Fig. 14).

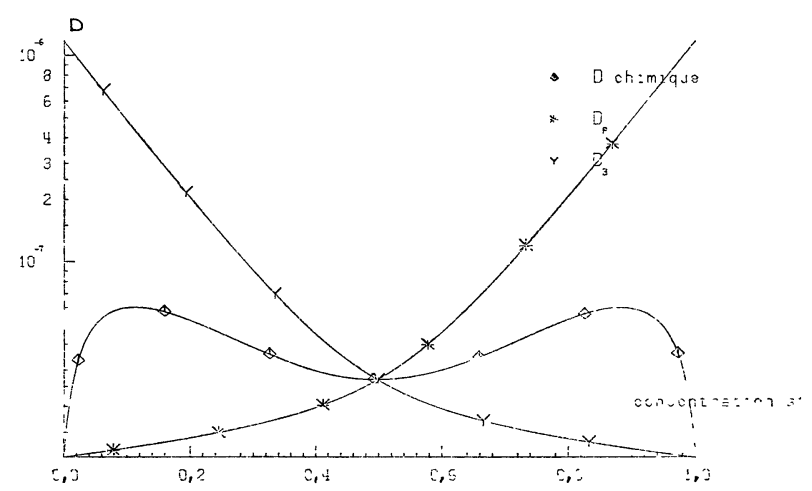

FIG. 14. - Coefficients $D_{\mathrm{A}}\left(N_{\mathrm{A}}\right), D_{\mathrm{B}}\left(N_{\mathrm{A}}\right)$ et $\tilde{D}\left(N_{\mathrm{A}}\right)$.

FIG. 14 à 17 . - Les figures 14 à 17 correspondent à un troisième essai du programme schématisé figure 1 , c'est pourquoi les graphiques sont élaborés sous la même forme : Cas des plans de Kirkendall multiples. $D_{\mathrm{A}}\left(N_{\mathrm{A}}\right)=D_{\mathrm{B}}\left(1-N_{\mathrm{A}}\right)$.

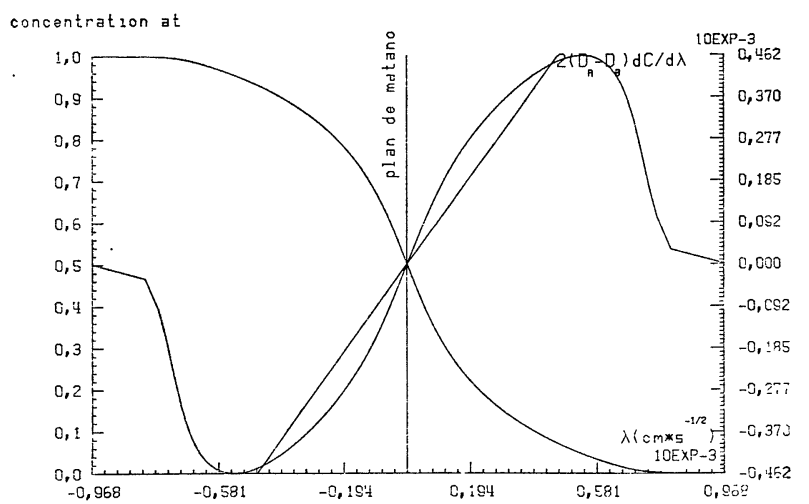

FIG. 15. - Profil de concentration $N_{\mathrm{A}}(\lambda)$ et vitesse réduite $F(\lambda)$.

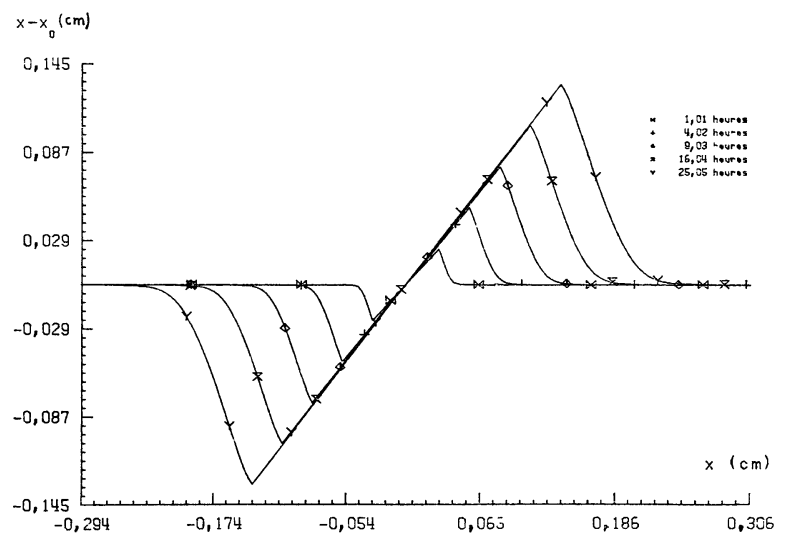

Fig. 16. - Profils de déplacement pour cinq durées de diffusion.

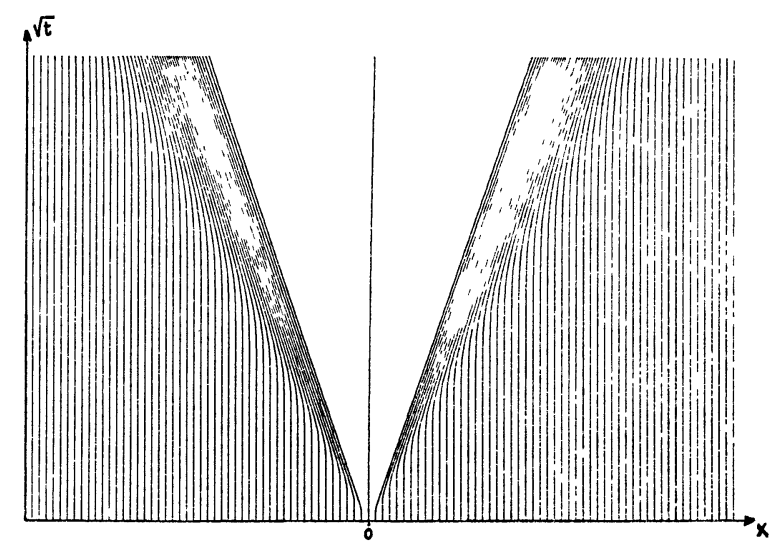

Fig. 17. - Diagramme des déplacements $\sqrt{t}(X)$.

Erratum: Ce qui est blanc est en réalité noir, s'il n'y avait pas de phénomène de solarisation à la reproduction.

La fonction $F$ coupe trois fois la $1^{\text {re }}$ bissectrice, pour des concentrations correspondantes égales à (Fig. 15) :

$$
\begin{aligned}
& 1^{\circ} 93 \% \text { pour } \lambda_{k_{1}}=-4,24 \times 10^{-4} \mathrm{~cm} . \mathrm{s}^{-1 / 2} \\
& 2^{\circ} 50 \% \text { pour } \lambda_{k_{2}}=0 \\
& 3^{\circ} 7 \% \text { pour } \lambda_{k_{3}}=+4,24 \times 10^{-4} \mathrm{~cm} \cdot \mathrm{s}^{-1 / 2} \text {. }
\end{aligned}
$$

La figure 16 montre les courbes de déplacement pour des durées de $1,4,9,16$ et 25 h.

Les courbes sont homothétiques. Ce sont en outre des segments de droite $\left(X-X_{0}=X\right.$, c'est-à-dire $X_{0}=0$ ) lorsque $X$ est compris entre

$$
\lambda_{k_{1}} \sqrt{t} \text { et } \lambda_{k_{3}} \sqrt{t} \text {. }
$$

Le graphe déplacement-abscisse initiale $X_{0}$ mettrait en évidence une discontinuité.

La figure 17 met en évidence les trois plans de Kirkendall. Dans sa partie centrale, la moitié de la zone de diffusion est « recristallisée » c'est-à-dire constituée par des plans cristallins créés dans le transport de matière qui accompagne l'effet Kirkendall (zone claire de la figure 15).

Il faut s'attendre dans un tel cas à une porosité très importante à chacune des extrémités de la zone de diffusion (parties sombres de la figure 17). 
2.3.4 Système Fe-Pd. - J. P. Gomez [6] a mesuré un certain nombre de valeurs des coefficients $D_{\mathrm{Fe}}$ et $D_{\mathrm{Pd}}$ dans le système fer-palladium, à $1100^{\circ} \mathrm{C}$, par la méthode des couples incrémentaux.

La figure 18 montre le lissage que nous avons réalisé (hyperboles) sur ces deux fonctions dans l'intervalle $30 \%-100 \%$ at $\mathrm{Fe}$.

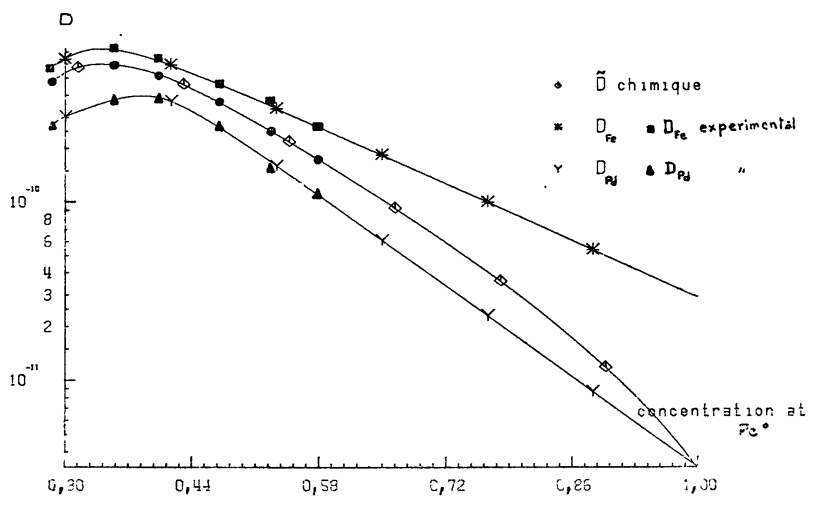

FIG. 18. - Coefficients de diffusion obtenus à partir d'un couple $\mathrm{Fe}$ pur/alliage Pd-Fe à $30 \%$ atomiques de fer.

La figure 19, présentant la fonction $F$, permet de prévoir pour un couple $30 \%-100 \% \mathrm{Fe}$ un plan de Kirkendall unique à la concentration de $58 \%$, le déplacement de ce plan après un recuit de $139 \mathrm{~h}$ étant de $33 \mu$.

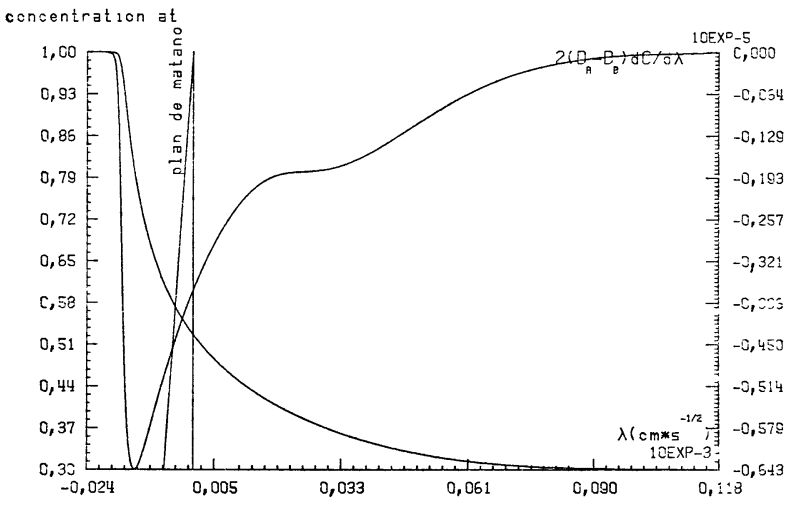

FIG. 19. - Profil de concentration et vitesse réduite $F(\eta)$.

Les valeurs expérimentales mesurées par Gomez sont respectivement $58,1 \% \pm 0,2 \%$ et $35 \mu \pm 5 \mu$.

La figure 20 met en évidence une dilatation de

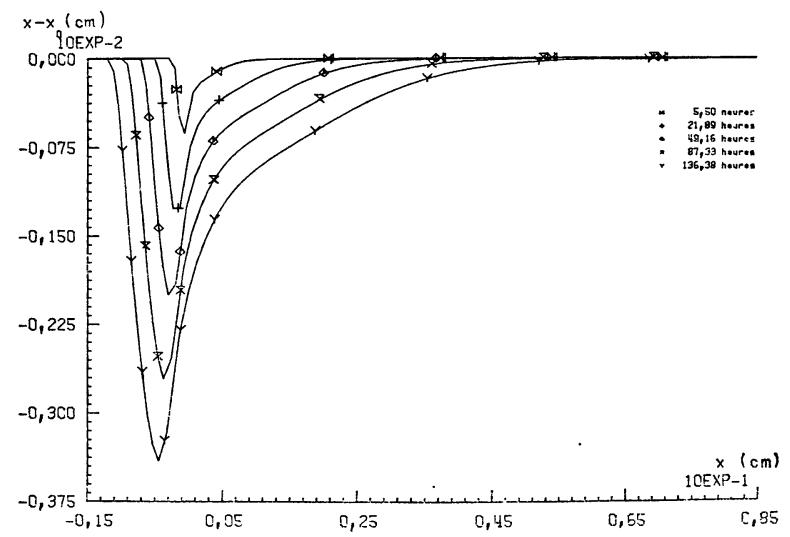

FIG. 20. - Profils de déplacement pour cinq durées de diffusion.

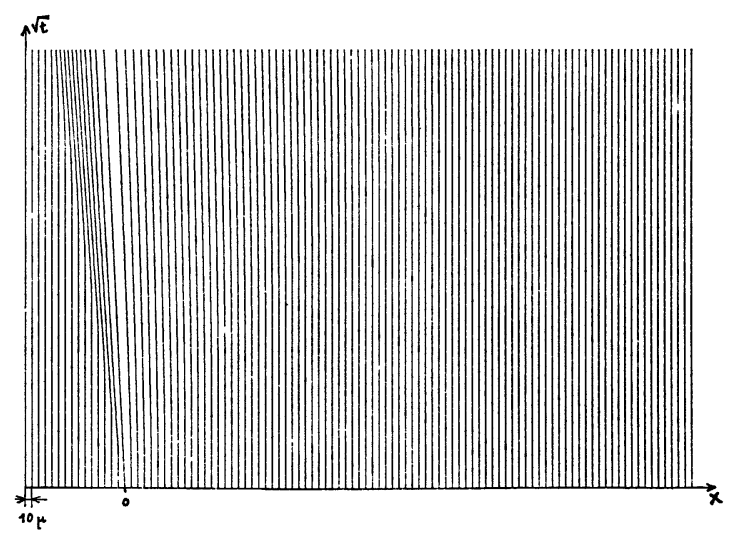

Fig. 21. - Diagramme des déplacements des plans cristallins en fonction de leur abscisse initial et du temps de recuit.

l'ordre de $100 \%$ du côté du fer pur, et de $50 \%$ du côté de l'alliage Pd-Fe $30 \%$.

Le diamètre des repères utilisés par Gomez est $15 \mu$ (fils de tungstène). Nous supposerons que les repères sont placés du côté de l'alliage Fe-Pd lors du collage du couple, qui est effectué sous pression.

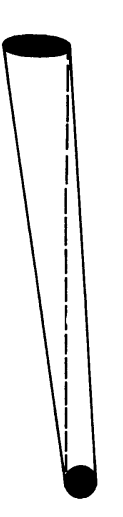

(a)

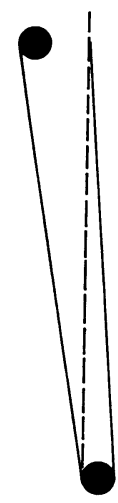

(b)

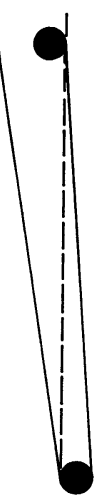

(c)

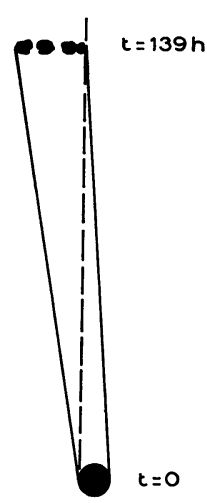

(d)
FIG. 22.

La figure $22 a$ montre quelle déformation devrait subir le fil de tungstène au cours du recuit de diffusion pour que la continuité des déplacements soit respectée. Le tungstène étant un métal réfractaire et assez fragile, ou bien il ne se déforme pas et le déplacement est un intermédiaire entre les cas $(22 b)$ et $(22 c)$, ou bien il y a fragmentation du fil $(22 d)$. Le cas $(22 d)$ a été observé dans un couple de diffusion par Gomez (Fig. 23).

Lorsqu'il n'y a pas fragmentation l'expérimentateur ne peut pas affirmer s'il est dans le cas $(22 b)$ ou (22c). Cela peut expliquer la dispersion, si souvent observée, mais pas toujours publiée, dans le déplacement des repères (fils de tungstène non alignés).

3. Problème inverse : programme « Kirkendall ». Les données sont sous forme de trois tableaux :

- un tableau d'abscisses $X$,

- un tableau de concentrations $C$ expérimentales correspondant aux abscisses $X$,

- un tableau de déplacements noté $X_{0}$; les déplacements sont ceux mesurés par la technique du mille- 

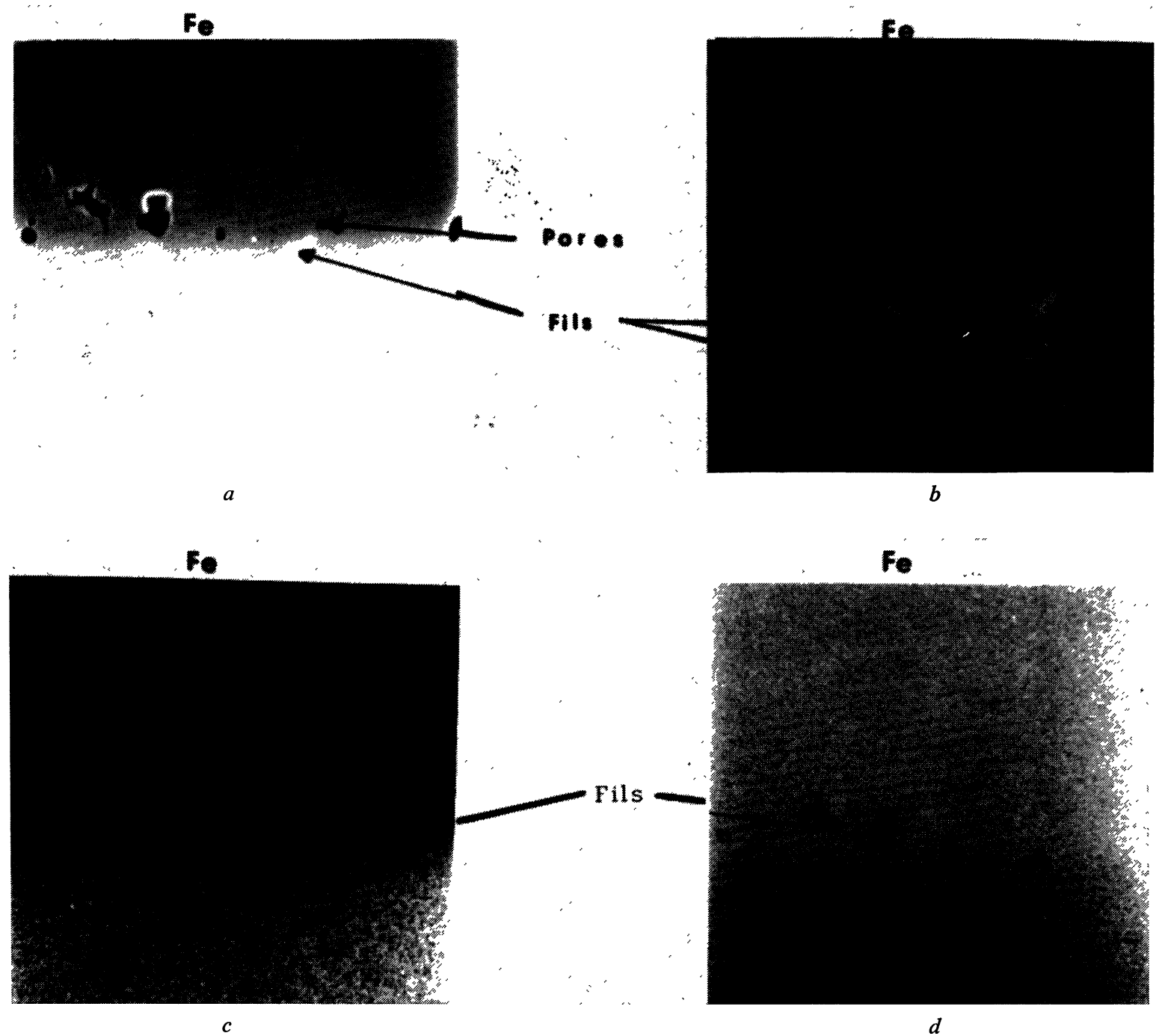

Fig. 23. - Observation par microanalyse $X$ de la zone de diffusion d'un couple Fe-Pd $30 \%$ Fe recuit à $1100^{\circ} \mathrm{C} 139 \mathrm{~h}: a$ ) image électronique, $b$ ) image $X$ du W, $c$ ) image du palladium, $d$ ) image $X$ du fer.

feuilles et correspondent aux abscisses $X$ où la concentration $C$ a été mesurée.

Les deux étapes essentielles du calcul sont :

$1^{\circ}$ Exploitation des tableaux $C$ et $X$ (concentrationpénétration) par la méthode de Matano. Le résultat est un tableau des coefficients de diffusion chimique $D$ correspondant aux concentrations expérimentales.

$2^{\circ}$ Exploitation des données $X$ et $X_{0}$ pour le calcul du rapport des coefficients intrinsèques $D_{\mathrm{A}} / D_{\mathrm{B}}$ par la formule de Heumann généralisée, formule (9), paragraphe 1.5 et :

$$
\frac{D_{\mathrm{A}}}{D_{\mathrm{B}}}=\frac{\int_{C_{\min }}^{C} X \mathrm{~d} C-C \cdot F \sqrt{t}}{\int_{C_{\min }}^{C} X \mathrm{~d} C+(1-C) F \sqrt{t}} .
$$

$3^{\circ}$ Résolution de l'équation

$$
D=C \cdot D_{\mathrm{B}}+(1-C) D_{\mathrm{A}}
$$

en chaque point, où $D, C$ et le rapport $D_{\mathrm{A}} / D_{\mathrm{B}}$ sont connus.

La sortie des résultats $\left(D_{\mathrm{A}}, D_{\mathrm{B}}, C\right)$ se fait sous forme graphique.

Un exemple est donné figure 24 dans le cas du système $\mathrm{Fe}-\mathrm{Pd}$.

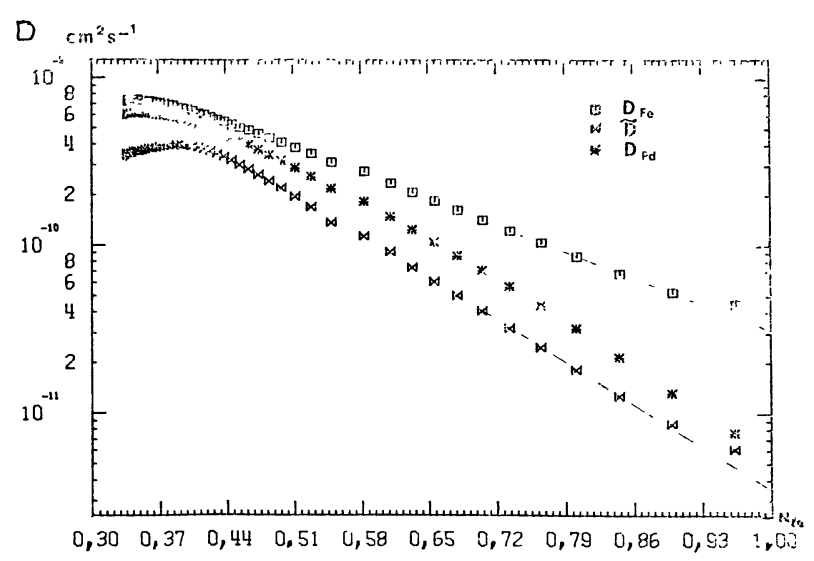

FIG. 24. 
La figure 3 donne un organigramme sommaire des calculs.

4. Vérification de la cohérence réciproque des deux programmes. - Cette vérification a pour but d'éliminer tous les doutes que l'on pourrait avoir sur la validité des calculs (erreurs de programmation, validité de la formule de Heumann généralisée).

La méthode consiste : à soumettre au programme Effet $\mathrm{K}$ un jeu de données arbitraires $D_{\mathrm{A}}, D_{\mathrm{B}}, C$, et à faire perforer, à l'issue des calculs du profil de concentration et des déplacements, un nouveau jeu de données pour le programme Kirkendall.

Ce dernier doit restituer, à la précision des calculs près $\left(10^{-3}\right)$ les fonctions $D_{\mathrm{A}}$ et $D_{\mathrm{B}}$ injectées initialement.

C'est ce que nous avons fait, en partant des valeurs $D_{\mathrm{Fe}}$ et $D_{\mathrm{Pd}}$ lissées à partir des données expérimentales de Gomez. Les données soumises sont celles de la figure 20 (déplacement-abscisse finale). Le résultat, figure 23, montre que la formule de Heumann généralisée restitue exactement les valeurs de $D_{\mathrm{Fe}}$ et $D_{\mathbf{P d}}$ données initialement.

5. Conclusion. - Les équations de Darken donnent sans aucun doute une description correcte de l'effet Kirkendall dans un couple de diffusion où il n'apparaîtrait pas de porosité ; dans la plupart des cas elles donnent une bonne approximation si la porosité n'est pas exagérément importante.

Les preuves en sont :

$1^{0}$ L'analyse de Matano en diffusion chimique est confirmée dans tous les systèmes présentant un effet Kirkendall, avec une bonne précision. Le coefficient de diffusion chimique $\widetilde{D}$ ne dépendant que de la concentration a donc une signification physique, indépendamment des considérations empiriques le liant aux propriétés thermodynamiques de l'alliage [7].

$2^{\circ}$ Le plan de Kirkendall se déplace en racine carrée du temps dans tous les systèmes où l'effet a été observé.

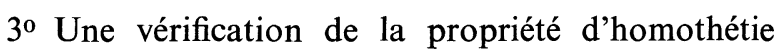
des courbes $x-x_{0}=f(x)$ a été établie dans le système $\mathrm{Ag}-\mathrm{Au} \mathrm{[8],} \mathrm{[3].}$

Il nous paraît donc opportun que désormais les expériences d'effet Kirkendall soient analysées dans le cadre de cette théorie et par les moyens que nous venons d'exposer, avant toute interprétation : facteur de corrélation, vent de lacunes...

Notre méthode, autocohérente, permet :

$1^{\circ}$ A partir d'une seule expérience d'effet Kirkendall (un seul temps de recuit à une température donnée pour un "mille-ieuilles " ou un couple avec interaces obliques repérés), de déterminer les valeurs des coefficients de diffusion intrinsèques dans tout l'intervalle de concentration.

$2^{\circ}$ A partir de plusieurs expériences effectuées à la même température (temps de recuit successifs), d'évaluer la précision des mesures et la validité des relations de Darken, par superposition des courbes de déplacement « réduites » (homothétie des courbes de déplacement).

\section{ANNEXE I}

Si le rapport $D_{\mathrm{A}} / D_{\mathrm{B}}=\alpha$ est constant, un extrémum de la fonction

$$
F=2\left(D_{\mathrm{A}}-D_{\mathrm{B}}\right) \frac{\mathrm{d} N_{\mathrm{A}}}{\mathrm{d} \lambda}
$$

coïncide avec le plan de Kirkendall.

En effet, dans tous les cas nous pouvons écrire :

$$
F=2(\alpha-1) D_{\mathbf{B}} \frac{\mathrm{d} N_{\mathrm{A}}}{\mathrm{d} \lambda}
$$

et

$$
\widetilde{D}=D_{\mathbf{B}}+(\alpha-1) N_{\mathbf{B}} D_{\mathbf{B}}
$$

soit :

$$
\widetilde{D} \frac{\mathrm{d} N_{\mathrm{A}}}{\mathrm{d} \lambda}=\frac{1}{2}\left(\frac{F}{\alpha-1}+N_{\mathrm{B}} F\right)
$$

L'équation de Fick donne alors :

$$
-\lambda \frac{\mathrm{d} N_{\mathrm{A}}}{\partial \lambda}=\frac{\mathrm{d}}{\mathrm{d} \lambda}\left(N_{\mathrm{B}} F+\frac{F}{\alpha-1}\right)
$$

ou

$$
\begin{aligned}
-\lambda \frac{\mathrm{d} N_{\mathrm{A}}}{\mathrm{d} \lambda}=\left(N_{\mathrm{B}}+\frac{1}{\alpha-1}\right) \frac{\mathrm{d} F}{\mathrm{~d} \lambda}+ & F \frac{\mathrm{d} N_{\mathrm{B}}}{\mathrm{d} \lambda}+ \\
& +F \frac{\mathrm{d}}{\mathrm{d} \lambda}\left(\frac{1}{\alpha-1}\right) .
\end{aligned}
$$

On posera

$$
K=\frac{1}{\alpha-1}=\frac{D_{\mathrm{B}}}{D_{\mathrm{A}}-D_{\mathrm{B}}}
$$

il vient :

$$
(F-\lambda) \frac{\mathrm{d} N_{\mathrm{A}}}{\mathrm{d} \lambda}=\left(K+N_{\mathrm{B}}\right) \frac{\mathrm{d} F}{\mathrm{~d} \lambda}+F \frac{\mathrm{d} K}{\mathrm{~d} \lambda}
$$

Notons que la quantité $K+N_{\text {B }}$ peut s'écrire :

$$
K+N_{\mathrm{B}}=\frac{D_{\mathrm{B}}}{D_{\mathrm{A}}-D_{\mathrm{B}}}+N_{\mathrm{B}}=\frac{\tilde{D}}{D_{\mathrm{A}}-D_{\mathrm{B}}} .
$$

Elle ne peut donc jamais être nulle.

Si le rapport $D_{\mathrm{A}} / D_{\mathrm{B}}$ est constant, $\mathrm{d} K / \mathrm{d} \lambda=0$.

Il vient :

$$
(F-\lambda) \frac{\mathrm{d} N_{\mathrm{A}}}{\mathrm{d} \lambda}=\left(K+N_{\mathrm{B}}\right) \frac{\mathrm{d} F}{\mathrm{~d} \lambda} .
$$

Au plan de Kirkendall,

$$
F=\lambda \quad \text { donc } \quad \frac{\mathrm{d} F}{\mathrm{~d} \lambda}=0
$$


Inversement, si au plan de Kirkendall la fonction $F$ présente un extrémum, la quantité $K$ doit aussi en présenter un (sauf si $F=0$ ); le rapport $D_{\mathrm{A}} / D_{\mathrm{B}}$ est donc lui aussi un extremum au plan de Kirkendall. Cela n'implique pas qu'il soit constant partout.

Adda et Philibert [4] montrent que dans ce cas les extrémums des flux $J_{\mathrm{A}}$ et $J_{\mathrm{B}}$ coïncident.

\section{ANNEXE II}

On désigne en théorie des champs par le mot « flux » (flux traversant une surface $(S)$ ) la quantité scalaire :

$$
\Phi=\iint_{X} \mathbf{J}_{\mathbf{i}} \mathrm{d} \mathbf{S}
$$

où $\mathbf{J}_{\mathbf{i}}$ est le vecteur du champ qui caractérise le transport.

Il est traditionnel, en diffusion, de désigner par « flux » le vecteur de transport lui-même.
Ce vecteur est défini en tout point du milieu comme la quantité :

$$
\mathbf{J}_{\mathbf{i}}=n_{\mathbf{i}} \mathbf{V}_{\mathbf{i}}
$$

où $n_{\mathbf{i}}$ est la «densité » d'atomes (nombre d'atomes par unité de volume) et $\mathbf{V}_{\mathbf{i}}$ la «vitesse moyenne» d'un atome $\mathrm{i}$ situé au point considéré.

La confusion est complète dans le cas qui nous intéresse (géométrie unidirectionnelle) où, par définition, le flux traversant une unité de surface perpendiculaire à la direction de diffusion s'écrit :

$$
\Phi=\iint_{S} \mathbf{J} \mathrm{d} \mathbf{S}=\mathbf{J} \cdot \mathbf{u}=\bar{J}
$$

$\mathbf{u}$ est le vecteur unitaire dans la direction de diffusion et $\bar{J}$ est la mesure algébrique du vecteur de transport sur cet axe.

Nous nous contenterons de signaler l'extension possible des formules au cas général de la diffusion dans une géométrie quelconque :

\section{ETABLISSEMENT DeS ÉQUATIONS DE LA DIFFUSiON ET DE L'EFFET KiRKeNDALL}

Tanslation : $\quad V=\frac{J_{\mathrm{A}}+J_{\mathrm{B}}}{n}$

Déformation : $\frac{\partial V}{\partial X}=\frac{1}{l} \frac{\mathrm{d} l}{\mathrm{~d} t}$

Conservation : $\frac{\partial N_{\mathrm{A}}}{\partial t}=\frac{1}{n} \frac{\partial}{X}\left(N_{\mathrm{B}} J_{\mathrm{A}}-N_{\mathrm{A}} J_{\mathrm{B}}\right)$

Fick :

$$
\left\{\begin{array}{l}
J_{\mathrm{A}}=-n D_{\mathrm{A}} \frac{\partial N_{\mathrm{A}}}{\partial X} \\
J_{\mathrm{B}}=-n D_{\mathrm{B}} \frac{\partial N_{\mathrm{B}}}{\partial X}
\end{array}\right.
$$

Fick + cons. $: \frac{\partial N_{\mathrm{A}}}{\partial t}=+\frac{\partial}{\partial X}\left[\left(N_{\mathrm{A}} D_{\mathrm{B}}+N_{\mathrm{B}} D_{\mathrm{A}}\right) \frac{\partial N_{\mathrm{A}}}{\partial X}\right]$

Déplacement des repères (valable seulement en géométrie unidirectionnelle). Définition

$$
F(\lambda)=2\left(D_{\mathrm{A}}-D_{\mathrm{B}}\right) \frac{\mathrm{d} N_{\mathrm{A}}}{\mathrm{d} \lambda} \text { avec } \lambda=\frac{X}{\sqrt{t}} .
$$

$X_{0}$ étant la position initiale du repère :

$$
\left.\left.V=\frac{\partial X}{\partial t}\right]_{X_{0}}=F(\lambda) \sqrt{t} \text { ou } \frac{\partial X}{\partial \sqrt{t}}\right]_{X_{0}}=F(\lambda)
$$

$$
\mathbf{V}=-\frac{\mathbf{J}_{\mathbf{A}}+\mathbf{J}_{\mathbf{B}}}{n}
$$

$\operatorname{div} \mathbf{V}=\frac{\mathrm{d} \varepsilon}{\mathrm{d} t}$

$$
\frac{\partial N_{\mathrm{A}}}{\partial t}=\frac{1}{n} \operatorname{div}\left(N_{\mathrm{B}} \mathbf{J}_{\mathrm{A}}-N_{\mathrm{A}} \mathbf{J}_{\mathrm{B}}\right)
$$$$
\mathbf{J}_{\mathrm{A}}=-n D_{\mathrm{A}} \operatorname{grad} \mathbf{N}_{\mathrm{A}}
$$

$$
\mathbf{J}_{\mathbf{B}}=-n D_{\mathbf{B}} \operatorname{grad} \mathbf{N}_{\mathbf{B}}
$$

$$
\left.\frac{\partial N_{\mathrm{A}}}{\partial t}=+\operatorname{div}\left[\left(N_{\mathrm{A}} D_{\mathrm{B}}+N_{\mathrm{B}} D_{\mathrm{A}}\right) \operatorname{grad} \mathbf{N}_{\mathrm{A}}\right)\right]
$$

$$
\left.\frac{\partial X}{\partial X_{0}}\right]_{\sqrt{ } \bar{t}}=\frac{\lambda-F}{\lambda_{0}} \text { avec } \lambda_{0}=\frac{X_{0}}{\sqrt{t}}, \quad X_{0} \neq 0
$$

pour $X_{0}=0$ (Kirkendall),

$$
\left.\lambda=F(\lambda) \quad \text { et } \quad \frac{\partial X}{\partial \sqrt{t}}\right]_{0}=\lambda=\frac{X}{\sqrt{t}} .
$$

\section{Bibliographie}

[1] CoRnet, J. F., Rapport CEA-R 4369, 1972.

[2] Connet, J. F., J. Phys. \& Chem. Solids 33 (1972) 1675.

[3] CoRnet, J. F., Thèse, Lyon, 16-1-73.

[4] Adda, Y. et Philibert, J., La diffusion dans les solides, Tome 1 (Presses Universitaires de France) 1966, 591.
[5] Adda, Y., Mairy, C., Andreu, J. L., Mem. Sci. Rev. Met. 61 (1964) 459.

[6] GomeZ, J. P. et RÉmy, C., Communication privée.

[7] Vignes, A. et Birchenall, C. E., Acta Met. 16 (1968) 1117, 25.

[8] Monty, C., Thèse, Orsay, juin 1972. 